Proceedings of the Edinburgh Mathematical Society (2003) 46, 279-292 (C)

DOI:10.1017/S0013091502000391 Printed in the United Kingdom

\title{
EXISTENCE OF POSITIVE SOLUTIONS FOR SUPERLINEAR SEMIPOSITONE $m$-POINT BOUNDARY-VALUE PROBLEMS
}

\author{
RUYUN MA \\ Department of Mathematics, Northwest Normal University, \\ Lanzhou 730070, Gansu, People's Republic of China (mary@nwnu.edu.cn)
}

(Received 18 April 2002)

Abstract In this paper we consider the existence of positive solutions to the boundary-value problems

$$
\begin{aligned}
\left(p(t) u^{\prime}\right)^{\prime}-q(t) u+\lambda f(t, u) & =0, \quad r<t<R, \\
a u(r)-b p(r) u^{\prime}(r) & =\sum_{i=1}^{m-2} \alpha_{i} u\left(\xi_{i}\right), \\
c u(R)+d p(R) u^{\prime}(R) & =\sum_{i=1}^{m-2} \beta_{i} u\left(\xi_{i}\right),
\end{aligned}
$$

where $\lambda$ is a positive parameter, $a, b, c, d \in[0, \infty), \xi_{i} \in(r, R), \alpha_{i}, \beta_{i} \in[0, \infty)$ (for $\left.i \in\{1, \ldots m-2\}\right)$ are given constants satisfying some suitable conditions. Our results extend some of the existing literature on superlinear semipositone problems. The proofs are based on the fixed-point theorem in cones.

Keywords: multipoint boundary-value problems; positive solutions; fixed-point theorem; cones

2000 Mathematics subject classification: Primary 34B10, 34B18, 34B15

\section{Introduction}

Multipoint boundary-value problems (BVPs) for ordinary differential equations arise in a variety of areas of applied mathematics and physics. For example, the vibrations of a guy wire of uniform cross-section and composed of $N$ parts of different densities can be set up as a multipoint BVP [7]; also, many problems in the theory of elastic stability can be handled by multipoint problems $[\mathbf{9}]$.

In [5], Il'in and Moiseev studied the existence of solutions for a linear multipoint BVP. Motivated by that study, Gupta [3] studied certain three-point BVPs for nonlinear ordinary differential equations. Since then, more general nonlinear multipoint BVPs have been studied by several authors. We refer the reader to $[\mathbf{3}, \mathbf{4}, \mathbf{6}, \mathbf{1 0}]$ for some references. 
In this paper, we are interested in the existence of positive solutions for the secondorder $m$-point BVP

$$
\left.\begin{array}{rl}
\left(p(t) u^{\prime}\right)^{\prime}-q(t) u+\lambda f(t, u) & =0, \quad r<t<R, \\
a u(r)-b p(r) u^{\prime}(r) & =\sum_{i=1}^{m-2} \alpha_{i} u\left(\xi_{i}\right), \\
c u(R)+d p(R) u^{\prime}(R) & =\sum_{i=1}^{m-2} \beta_{i} u\left(\xi_{i}\right),
\end{array}\right\}
$$

where $p, q \in C([r, R],(0, \infty)), a, b, c, d \in[0, \infty), \xi_{i} \in(Y, R), \alpha_{i}, \beta_{i} \in(0, \infty)$ (for $i \in$ $\{1, \ldots m-2\})$ are given constants. If $q \equiv 0$ and $\alpha_{i}=\beta_{i}=0$ for $i=1, \ldots, m-2$, then the $m$-point BVP (1.1) reduces to the two-point BVP

$$
\left.\begin{array}{rl}
\left(p(t) u^{\prime}\right)^{\prime}+\lambda f(t, u) & =0, \quad r<t<R, \\
a u(r)-b p(r) u^{\prime}(r) & =0, \\
c u(R)+d p(R) u^{\prime}(R) & =0 .
\end{array}\right\}
$$

In 1996, Anuradha, Hai and Shivaji [1] studied the existence of positive solutions for (1.2) under the assumptions:

(A1) $p \in C([r, R],(0, \infty))$;

(A2) $a, b, c, d \in[0, \infty)$ with $a c+a d+b c>0$;

(A3) $f:[r, R] \times[0, \infty) \rightarrow R$ is continuous and there exists an $M>0$ such that $f(t, u) \geqslant-M$ for every $t \in[r, R], u \geqslant 0$; and

(A4) $\lim _{u \rightarrow \infty}(f(t, u) / u)=\infty$ uniformly on a compact subinterval $[\alpha, \beta]$ of $(r, R)$.

They established the following result for (1.2).

Theorem 1.1 (see Theorem 1 in [1]). Suppose that (A1)-(A4) hold. Then (1.2) has a positive solution for $\lambda>0$ sufficiently small.

If $r=0, R=1, \lambda=1, p(t) \equiv 1, q(t) \equiv 0, f(t, u)=h(t) \bar{f}(u), a=c=1, b=d=0$, $\alpha_{i}=0$ for $i=1, \ldots, m-2$, and $\beta_{j}=0$ for $j=2, \ldots, m-2$, then (1.1) reduces to the three-point BVP

$$
\left.\begin{array}{c}
u^{\prime \prime}+h(t) \bar{f}(u)=0, \quad 0<t<1, \\
u(0)=0, \quad u(1)=\beta_{1} u\left(\xi_{1}\right) .
\end{array}\right\}
$$

In 1999, Ma [6] obtained the following result for (1.3).

Theorem 1.2 (see Theorem 1 in [6]).

(H1) $0<\beta_{1} \xi_{1}<1$.

(H2) $\bar{f} \in C([0, \infty),[0, \infty))$.

(H3) $h \in C([0,1],[0, \infty))$ and there exists $t_{0} \in\left[\xi_{1}, 1\right]$ such that $h\left(t_{0}\right)>0$. 
Then (1.3) has at least one positive solution in one of the two following cases:

(i) $\bar{f}_{0}=0$ and $\bar{f}_{\infty}=\infty$,

(ii) $\bar{f}_{0}=\infty$ and $\bar{f}_{\infty}=0$,

where

$$
\bar{f}_{0}:=\lim _{u \rightarrow 0^{+}} \frac{\bar{f}(u)}{u}, \quad \bar{f}_{\infty}:=\lim _{u \rightarrow \infty} \frac{\bar{f}(u)}{u} .
$$

Theorem 1.2 has been improved by Webb [10]. We remark that in the proof of Theorem 1.2 we rewrite (1.3) as the following equivalent integral equation:

$$
\begin{aligned}
u(t)=-\int_{0}^{t}(t-s) h(s) \bar{f}(u(s)) \mathrm{d} s- & \frac{\beta_{1} t}{1-\beta_{1} \xi_{1}} \int_{0}^{\xi_{1}}\left(\xi_{1}-s\right) h(s) \bar{f}(u(s)) \mathrm{d} s \\
& +\frac{t}{1-\beta_{1} \xi_{1}} \int_{0}^{1}(1-s) h(s) \bar{f}(u(s)) \mathrm{d} s \\
:=(A u)(t) . &
\end{aligned}
$$

Clearly, $(A u)(t)$ contains one positive term and two negative terms. This form is not convenient for studying the existence of positive solutions. In fact, in order to apply the fixed-point theorem in cones, we need to show that

$$
(A y)(t) \geqslant 0, \quad \text { for all } y \in C([0,1],[0, \infty)) \text { and } t \in[0,1] .
$$

Since $A y$ contains two negative terms, it is not easy to show that (H1)-(H3) imply that (1.5) holds.

In this paper, we consider the more general $m$-point BVP (1.1). To deal with (1.1), we give a new integral equation which is equivalent to

$$
\begin{aligned}
\left(p(t) u^{\prime}\right)^{\prime}-q(t) u+y(t) & =0, \quad r<t<R, \\
a u(r)-b p(r) u^{\prime}(r) & =\sum_{i=1}^{m-2} \alpha_{i} u\left(\xi_{i}\right), \\
c u(R)+d p(R) u^{\prime}(R) & =\sum_{i=1}^{m-2} \beta_{i} u\left(\xi_{i}\right),
\end{aligned}
$$

and contains two positive terms if $y \geqslant 0$. Our most important result (see Theorem 3.1 below) extends the main results of $[\mathbf{1}]$ in two directions:

(i) the $m$-point BVP (1.1) is considered; and

(ii) the case $q(t)>0$ is studied.

By a positive solution of (1.1) we understand a function $u(t)$ which is positive on $(r, R)$ and satisfies the differential equation and the boundary conditions in (1.1).

The main tool of this paper is the following well-known Guo-Krasnoselskii fixed-point theorem. 
Theorem 1.3 (see [2]). Let $E$ be a Banach space, and let $K \subset E$ be a cone. Assume $\Omega_{1}, \Omega_{2}$ are open bounded subsets of $E$ with $0 \in \Omega_{1}, \bar{\Omega}_{1} \subset \Omega_{2}$, and let

$$
A: K \cap\left(\bar{\Omega}_{2} \backslash \Omega_{1}\right) \rightarrow K
$$

be a completely continuous operator such that

(i) $\|A u\| \leqslant\|u\|, u \in K \cap \partial \Omega_{1}$, and $\|A u\| \geqslant\|u\|, u \in K \cap \partial \Omega_{2}$; or

(ii) $\|A u\| \geqslant\|u\|, u \in K \cap \partial \Omega_{1}$, and $\|A u\| \leqslant\|u\|, u \in K \cap \partial \Omega_{2}$.

Then $A$ has a fixed point in $K \cap\left(\bar{\Omega}_{2} \backslash \Omega_{1}\right)$.

\section{Preliminary lemmas}

In the rest of the paper, we make the following assumptions:

(C1) $p \in C^{1}([r, R],(0, \infty)), q \in C([r, R],(0, \infty))$; and

(C2) $a, b, c, d \in[0, \infty)$ with $a c+a d+b c>0, \alpha_{i}, \beta_{i} \in[0, \infty)$ for $i \in\{1, \ldots m-2\}$.

To state and prove the main results of this paper, we need the following lemmas.

Lemma 2.1. Let (C1) and (C2) hold. Let $\psi$ and $\phi$ be the solutions of the linear problems

$$
\left.\begin{array}{c}
\left(p(t) \psi^{\prime}(t)\right)^{\prime}-q(t) \psi(t)=0, \\
\psi(r)=b, \quad p(r) \psi^{\prime}(r)=a
\end{array}\right\}
$$

and

$$
\left.\begin{array}{c}
\left(p(t) \phi^{\prime}(t)\right)^{\prime}-q(t) \phi(t)=0, \\
\phi(R)=d, \quad p(R) \phi^{\prime}(R)=-c,
\end{array}\right\}
$$

respectively. Then

(i) $\psi$ is strictly increasing on $[r, R]$, and $\psi(t)>0$ on $(r, R]$; and

(ii) $\phi$ is strictly decreasing on $[r, R]$, and $\phi(t)>0$ on $[r, R)$.

Proof. We shall give a proof for (i) only. The proof of (ii) follows in a similar manner. It is easy to see that (2.1) is equivalent to the problem

$$
\left.\begin{array}{c}
\psi^{\prime \prime}(t)+\frac{p^{\prime}(t)}{p(t)} \psi^{\prime}(t)-\frac{q(t)}{p(t)} \psi(t)=0, \\
\psi(r)=b, \quad \psi^{\prime}(r)=\frac{a}{p(r)} .
\end{array}\right\}
$$

Now we divide the proof into three steps. 
Step 1. We show that there exists $\sigma \in(0, R-r)$ such that $\psi$ is strictly increasing on $(r, r+\sigma)$.

If $a>0$, then we are done. If $a=0$, then we know from (C2) that $b>0$. Therefore, we have from (2.3) that

$$
\psi^{\prime \prime}(r)=\frac{q(r)}{p(r)} \psi(r)>0,
$$

which implies that there exists $\sigma>0$ such that $\psi^{\prime}(t)>0$ on $(r, r+\sigma)$. Thus $\psi(t)$ is strictly increasing on $(r, r+\sigma)$.

Step 2. We show that $\psi$ has no local maxima on all of $(r, R)$.

In fact, by Step $1, \psi$ is positive and strictly increasing on $(r, r+\sigma)$. So we can apply the maximum principle (see [8, Theorem 1 of Chapter 1$]$ ) to show that there are no local maxima on $(r, R)$. Moreover, $\psi$ is non-decreasing on $(r, R)$.

Step 3. We show that $\psi$ is strictly increasing on $[r, R]$.

If there exists $t_{2}, t_{3} \subset[r, R]$ with $t_{2}<t_{3}$ such that $\psi\left(t_{2}\right)=\psi\left(t_{3}\right)$, then

$$
\psi(t) \equiv \psi\left(t_{3}\right), \quad t \in\left[t_{2}, t_{3}\right] .
$$

This implies

$$
\psi^{\prime}(t)=\psi^{\prime \prime}(t)=0, \quad t \in\left[t_{2}, t_{3}\right] .
$$

We note that by Steps 1 and $2, \psi\left(t_{3}\right)>0$. Thus from (2.3) we get

$$
\psi^{\prime \prime}\left(t_{3}\right)=\frac{q\left(t_{3}\right)}{p\left(t_{3}\right)} \psi\left(t_{3}\right)>0 .
$$

This contradicts the fact that $\psi^{\prime \prime}\left(t_{3}\right)=0$.

Notation. Set

$$
\rho:=p(r)\left|\begin{array}{cc}
\phi(r) & \psi(r) \\
\phi^{\prime}(r) & \psi^{\prime}(r)
\end{array}\right|, \quad \Delta:=\left|\begin{array}{cc}
-\sum_{i=1}^{m-2} \alpha_{i} \psi\left(\xi_{i}\right) & \rho-\sum_{i=1}^{m-2} \alpha_{i} \phi\left(\xi_{i}\right) \\
\rho-\sum_{i=1}^{m-2} \beta_{i} \psi\left(\xi_{i}\right) & -\sum_{i=1}^{m-2} \beta_{i} \phi\left(\xi_{i}\right)
\end{array}\right| .
$$

Lemma 2.2. Let (C1) and (C2) hold. Assume that

(C3) $\Delta: \neq 0$.

Then for $y \in C[r, R]$, the problem

$$
\left.\begin{array}{rl}
\left(p(t) u^{\prime}(t)\right)^{\prime}-q(t) u(t)+y(t) & =0, \quad r<t<R, \\
a u(r)-b u^{\prime}(r) & =\sum_{i=1}^{m-2} \alpha_{i} u\left(\xi_{i}\right), \\
c u(R)+d u^{\prime}(R) & =\sum_{i=1}^{m-2} \beta_{i} u\left(\xi_{i}\right)
\end{array}\right\}
$$


has a unique solution

$$
u(t)=\int_{r}^{R} G(t, s) y(s) \mathrm{d} s+A(y) \psi(t)+B(y) \phi(t),
$$

where

$$
\begin{gathered}
G(t, s)=\frac{1}{\rho} \begin{cases}\phi(t) \psi(s), & r \leqslant s \leqslant t \leqslant R, \\
\phi(s) \psi(t), & r \leqslant t \leqslant s \leqslant R,\end{cases} \\
A(y):=\frac{1}{\Delta}\left|\begin{array}{ll}
\sum_{i=1}^{m-2} \alpha_{i} \int_{r}^{R} G\left(\xi_{i}, s\right) y(s) \mathrm{d} s & \rho-\sum_{i=1}^{m-2} \alpha_{i} \phi\left(\xi_{i}\right) \\
\sum_{i=1}^{m-2} \beta_{i} \int_{r}^{R} G\left(\xi_{i}, s\right) y(s) \mathrm{d} s & -\sum_{i=1}^{m-2} \beta_{i} \phi\left(\xi_{i}\right)
\end{array}\right|
\end{gathered}
$$

and

$$
B(y):=\frac{1}{\Delta}\left|\begin{array}{ll}
-\sum_{i=1}^{m-2} \alpha_{i} \psi\left(\xi_{i}\right) & \sum_{i=1}^{m-2} \alpha_{i} \int_{r}^{R} G\left(\xi_{i}, s\right) y(s) \mathrm{d} s \\
\rho-\sum_{i=1}^{m-2} \beta_{i} \psi\left(\xi_{i}\right) & \sum_{i=1}^{m-2} \beta_{i} \int_{r}^{R} G\left(\xi_{i}, s\right) y(s) \mathrm{d} s
\end{array}\right| .
$$

Proof. The proof follows by routine calculations.

Lemma 2.3. Let (C1) and (C2) hold. Assume

(C4) $\Delta<0, \rho-\sum_{i=1}^{m-2} \alpha_{i} \phi\left(\xi_{i}\right)>0, \rho-\sum_{i=1}^{m-2} \beta_{i} \psi\left(\xi_{i}\right)>0$.

Then for $y \in C[r, R]$ with $y \geqslant 0$, the unique solution $u$ of the problem (2.4) satisfies

$$
u(t) \geqslant 0, \quad \text { for } t \in[r, R]
$$

Proof. This is an immediate consequence of the facts that $G \geqslant 0$ on $[r, R] \times[r, R]$ and

$$
A(y) \geqslant 0, \quad B(y) \geqslant 0
$$

Lemma 2.4. Let (C1), (C2) and (C4) hold. Let

$$
\tilde{q}(t):=\min \left\{\frac{\phi(t)}{\phi(r)}, \frac{\psi(t)}{\psi(R)}\right\} .
$$

Then for $y \in C[r, R]$ with $y \geqslant 0$, the unique solution $u$ of the problem (2.4) satisfies

$$
u(t) \geqslant \frac{1}{2} \gamma(t)\|u\|
$$


where $\|u\|=\max \{u(t) \mid t \in[r, R]\}$ and

$$
\gamma(t):=\frac{1}{k_{0}}[\tilde{q}(t)+\tilde{A} \psi(t)+\tilde{B} \phi(t)]
$$

with $k_{0} \in N$ a fixed integer such that

$$
\begin{gathered}
\frac{1}{k_{0}}[\tilde{q}(t)+\tilde{A} \psi(t)+\tilde{B} \phi(t)] \leqslant 1, \quad \text { for all } t \in[r, R], \\
\tilde{A}:=\frac{1}{\Delta}\left|\begin{array}{ll}
\sum_{i=1}^{m-2} \alpha_{i} \tilde{q}\left(\xi_{i}\right) & \rho-\sum_{i=1}^{m-2} \alpha_{i} \phi\left(\xi_{i}\right) \\
\sum_{i=1}^{m-2} \beta_{i} \tilde{q}\left(\xi_{i}\right) & -\sum_{i=1}^{m-2} \beta_{i} \phi\left(\xi_{i}\right)
\end{array}\right|
\end{gathered}
$$

and

$$
\tilde{B}:=\frac{1}{\Delta}\left|\begin{array}{cc}
-\sum_{i=1}^{m-2} \alpha_{i} \psi\left(\xi_{i}\right) & \sum_{i=1}^{m-2} \alpha_{i} \tilde{q}\left(\xi_{i}\right) \\
\rho-\sum_{i=1}^{m-2} \beta_{i} \psi\left(\xi_{i}\right) & \sum_{i=1}^{m-2} \beta_{i} \tilde{q}\left(\xi_{i}\right)
\end{array}\right|
$$

Proof. We have from (2.6) that

$$
0 \leqslant G(t, s) \leqslant G(s, s), \quad t \in[r, R]
$$

which implies

$$
u(t) \leqslant \int_{r}^{R} G(s, s) y(s) \mathrm{d} s+A(y) \psi(t)+B(y) \phi(t), \quad \text { for all } t \in[r, R] .
$$

Applying (2.6), we have that for $t \in[r, R]$

$$
\begin{aligned}
\frac{G(t, s)}{G(s, s)}= \begin{cases}\frac{\phi(t)}{\phi(s)}, & r \leqslant s \leqslant t \leqslant R, \\
\frac{\psi(t)}{\psi(s)}, & r \leqslant t \leqslant s \leqslant R,\end{cases} \\
\geqslant \begin{cases}\frac{\phi(t)}{\phi(r)}, & r \leqslant s \leqslant t \leqslant R, \\
\frac{\psi(t)}{\psi(R)}, & r \leqslant t \leqslant s \leqslant R,\end{cases} \\
\geqslant \tilde{q}(t),
\end{aligned}
$$

where $\tilde{q}(t)$ is as in (2.11). Combining (2.16) with (2.7) and (2.8), we can conclude that

$$
A(y) \geqslant \tilde{A} \int_{r}^{R} G(s, s) y(s) \mathrm{d} s, \quad B(y) \geqslant \tilde{B} \int_{r}^{R} G(s, s) y(s) \mathrm{d} s
$$


where $\tilde{A}$ and $\tilde{B}$ are as in (2.13) and (2.14), respectively. Thus for $t \in[r, R]$,

$$
\begin{aligned}
u(t) & =\int_{r}^{R} G(t, s) y(s) \mathrm{d} s+A(y) \psi(t)+B(y) \phi(t) \\
& \geqslant \frac{1}{2}\left[\int_{r}^{R} G(t, s) y(s) \mathrm{d} s+A(y) \psi(t)+B(y) \phi(t)\right]+\frac{1}{2}[A(y) \psi(t)+B(y) \phi(t)] \\
& =\frac{1}{2}\left[\int_{r}^{R} \frac{G(t, s)}{G(s, s)} G(s, s) y(s) \mathrm{d} s+A(y) \psi(t)+B(y) \phi(t)\right]+\frac{1}{2}[A(y) \psi(t)+B(y) \phi(t)] \\
& \geqslant \frac{1}{2}\left[\tilde{q}(t) \int_{r}^{R} G(s, s) y(s) \mathrm{d} s+\tilde{A} \int_{r}^{R} G(s, s) y(s) \mathrm{d} s \psi(t)\right. \\
& =\frac{1}{2} k_{0} \gamma(t) \int_{r}^{R} G(s, s) y(s) \mathrm{d} s+\frac{1}{2}[A(y) \psi(t)+B(y) \phi(t)] \\
& \geqslant \frac{1}{2} \gamma(t)\left[\int_{r}^{R} G(s, s) y(s) \mathrm{d} s+A(y) \psi(t)+B(y) \phi(t)\right] \\
& \geqslant \frac{1}{2} \gamma(t)\|u\| \quad(b y(2.15)),
\end{aligned}
$$

where

$$
\gamma(t):=\frac{1}{k_{0}}[\tilde{q}(t)+\tilde{A} \psi(t)+\tilde{B} \phi(t)]
$$

Lemma 2.5. Let (C1)-(C4) hold and Let $\bar{w}$ be the solution of

$$
\left.\begin{array}{rl}
\left(p(t) u^{\prime}(t)\right)^{\prime}-q(t) u(t)+1 & =0, \quad r<t<R, \\
a u(r)-b u^{\prime}(r) & =\sum_{i=1}^{m-2} \alpha_{i} u\left(\xi_{i}\right), \\
c u(R)+d u^{\prime}(R) & =\sum_{i=1}^{m-2} \beta_{i} u\left(\xi_{i}\right) .
\end{array}\right\}
$$

Then there exists a positive number $C$ such that $\bar{w}(t) \leqslant C \gamma(t)$ for every $t \in[r, R]$.

Proof. By Lemma 2.2, we know that

$$
\begin{aligned}
\bar{w}(t) & =\int_{r}^{R} G(t, s) \mathrm{d} s+A(1) \psi(t)+B(1) \phi(t) \\
& =\frac{1}{\rho}\left[\int_{r}^{t} \phi(t) \psi(s) \mathrm{d} s+\int_{r}^{t} \psi(t) \phi(s) \mathrm{d} s\right]+A(1) \psi(t)+B(1) \phi(t)
\end{aligned}
$$




$$
\begin{aligned}
& \leqslant \frac{1}{\rho}\left[\int_{r}^{t} \phi(t) \psi(t) \mathrm{d} s+\int_{t}^{R} \psi(t) \phi(t) \mathrm{d} s\right]+A(1) \psi(t)+B(1) \phi(t) \\
& \leqslant \frac{1}{\rho}(R-r) \phi(t) \psi(t)+A(1) \psi(t)+B(1) \phi(t) \\
& \leqslant \frac{1}{\rho}(R-r) \phi(r) \psi(R) \tilde{q}(t)+A(1) \psi(t)+B(1) \phi(t) \\
& =\frac{1}{\rho}(R-r) \phi(r) \psi(R) \tilde{q}(t)+\frac{A(1)}{\tilde{A}} \tilde{A} \psi(t)+\frac{B(1)}{\tilde{B}} \tilde{B} \phi(t) \\
& \leqslant \mu[\tilde{q}(t)+\tilde{A} \psi(t)+\tilde{B} \phi(t)] \\
& =C \gamma(t)
\end{aligned}
$$

where $C:=k_{0} \mu$ and

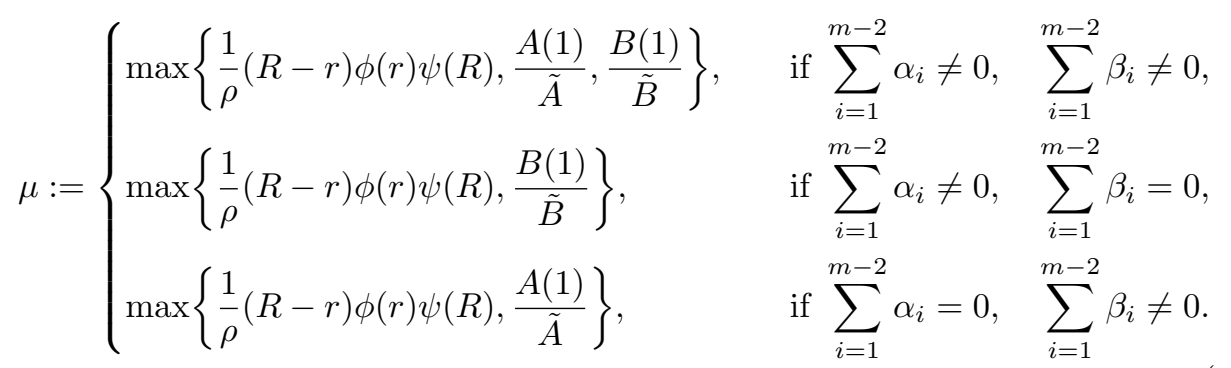

We note that

$$
\tilde{A}>0 \quad \text { if } \sum_{i=1}^{m-2} \beta_{i} \neq 0
$$

and

$$
\tilde{B}>0 \quad \text { if } \sum_{i=1}^{m-2} \alpha_{i} \neq 0 .
$$

So the constant $C$ in (2.19) is well defined.

\section{The main result}

The main result of the paper is the following theorem.

Theorem 3.1. Let (C1), (C2), (C4) and (A3) and (A4) hold. Then (1.1) has a positive solution for $\lambda>0$ sufficiently small.

Remark 3.2. Theorem 3.1 extends [1, Theorem 1] in two main directions:

(i) the $m$-point BVPs (1.1) are considered; and

(ii) the case $q(t)>0$ is studied. 
Proof of Theorem 3.1. Let $\lambda$ satisfy

$$
0<\lambda<\min \left\{\frac{1}{C_{1}\|\bar{w}\|}, \frac{1}{2 C M}\right\},
$$

where $C_{1}=\sup \{g(t, u) \mid r \leqslant t \leqslant R, 0 \leqslant u \leqslant 1\}, g(t, u):=f(t, u)+M$ and $C$ is the constant defined in Lemma 2.5. Let $w=\lambda M \bar{w}$. Then $u$ is a positive solution of (1.1) if and only if $\tilde{u}=u+w$ is a solution of

$$
\left.\begin{array}{rl}
\left(p(t) u^{\prime}\right)^{\prime}-q(t) u+\lambda \tilde{g}(t, u-w) & =0, \quad r<t<R, \\
a u(r)-b p(r) u^{\prime}(r) & =\sum_{i=1}^{m-2} \alpha_{i} u\left(\xi_{i}\right), \\
c u(R)+d p(R) u^{\prime}(R) & =\sum_{i=1}^{m-2} \beta_{i} u\left(\xi_{i}\right),
\end{array}\right\}
$$

with $\tilde{u}(t)>w(t)$ on $(r, R)$. Here

$$
\tilde{g}(t, u)= \begin{cases}g(t, u), & \text { for } u \geqslant 0, \\ g(t, 0), & \text { for } u<0 .\end{cases}
$$

Let

$$
K=\left\{u \in C[r, R]: u(t) \geqslant \frac{1}{2} \gamma(t)\|u\|, t \in[r, R]\right\},
$$

where $\gamma$ is as in (2.12). For each $v \in K$, let $u=T v$ be the solution of

$$
\left.\begin{array}{rl}
\left(p(t) u^{\prime}\right)^{\prime}-q(t) u+\lambda \tilde{g}(t, v-w) & =0, \quad r<t<R, \\
a u(r)-b p(r) u^{\prime}(r) & =\sum_{i=1}^{m-2} \alpha_{i} u\left(\xi_{i}\right), \\
c u(R)+d p(R) u^{\prime}(R) & =\sum_{i=1}^{m-2} \beta_{i} u\left(\xi_{i}\right) .
\end{array}\right\}
$$

By Lemma 2.2,

$$
T v=\lambda\left[\int_{r}^{R} G(t, s) \tilde{g}(s, v(s)-w(s)) \mathrm{d} s+A(\tilde{g}(\cdot, v-w)) \psi(t)+B(\tilde{g}(\cdot, v-w)) \phi(t)\right] .
$$

From Lemma 2.4, we know that $T: K \rightarrow K$. It is easy to check that $T$ is completely continuous. We shall prove that $T$ has a fixed point in $K$ by using Theorem 1.3.

Define $\Omega_{1}=\{u \in C[r, R]:\|u\|<1\}$. For $u \in \partial \Omega_{1} \cap K$,

$$
\begin{aligned}
(T v)(t) & =\lambda\left[\int_{r}^{R} G(t, s) \tilde{g}(s, v(s)-w(s)) \mathrm{d} s+A(\tilde{g}(\cdot, v-w)) \psi(t)+B(\tilde{g}(\cdot, v-w)) \phi(t)\right] \\
& \leqslant \lambda C_{1}\left[\int_{r}^{R} G(t, s) \mathrm{d} s+A(1) \psi(t)+B(1) \phi(t)\right] \\
& =\lambda C_{1} \bar{w}(t) \\
& \leqslant 1,
\end{aligned}
$$


since $0 \leqslant v-w \leqslant v \leqslant 1$. Thus

$$
\|T u\| \leqslant\|u\|, \quad \text { for } u \in \partial \Omega_{1} \cap K .
$$

Now choose a constant $\tilde{M}>0$ such that

$$
1 \leqslant \frac{1}{4} \lambda \tilde{M} \Gamma \inf _{r \leqslant t \leqslant R} \int_{\alpha}^{\beta} G(t, s) \mathrm{d} s,
$$

where

$$
\Gamma:=\min _{\alpha \leqslant t \leqslant \beta} \gamma(t) .
$$

By (A4), we know that there is a constant $D>0$ such that

$$
\frac{\tilde{g}(t, s)}{s} \geqslant \tilde{M}, \quad \text { for }(t, s) \in[\alpha, \beta] \times[D, \infty) .
$$

Set

$$
\rho_{2}=\max \left\{4,4 \lambda C M, \frac{4 D}{\Gamma}\right\}
$$

and define

$$
\Omega_{2}=\left\{u \in C[r, R]:\|u\|<\rho_{2}\right\} .
$$

For $u \in \partial \Omega_{2} \cap K$, we have from Lemmas 2.5 and 2.4 that

$$
\begin{aligned}
u(s)-w(s) & =u(s)-\lambda M \bar{w}(s) \\
& \geqslant u(s)-\lambda M C \gamma(s) \\
& \geqslant u(s)-\frac{\lambda C M}{\rho_{2}} 2 u(s) \\
& \geqslant \frac{1}{2} u(s)
\end{aligned}
$$

and

$$
\begin{aligned}
\min _{\alpha \leqslant s \leqslant \beta}(u(s)-w(s)) & \geqslant \min _{\alpha \leqslant s \leqslant \beta} \frac{1}{2} u(s) \\
& \geqslant \min _{\alpha \leqslant s \leqslant \beta} \frac{1}{4}\|u\| \gamma(s) \\
& =\frac{1}{2} \rho_{2} \Gamma \geqslant D .
\end{aligned}
$$

Therefore, for $u \in \partial \Omega_{2} \cap K$, we have

$$
\begin{aligned}
\min _{t \in[\alpha, \beta]}(T u)(t) & =\lambda \min _{t \in[\alpha, \beta]} \int_{r}^{R} G(t, s) \tilde{g}(s, u-w) \mathrm{d} s \\
& \quad+A(\tilde{g}(\cdot, u-w)) \psi(t)+B(\tilde{g}(\cdot, u-w)) \phi(t) \\
& \geqslant \lambda \min _{t \in[\alpha, \beta]} \int_{r}^{R} G(t, s) \tilde{g}(s, u-w) \mathrm{d} s
\end{aligned}
$$




$$
\begin{aligned}
& \geqslant \lambda \min _{t \in[\alpha, \beta]} \int_{r}^{R} G(t, s) \tilde{M}(u(s)-w(s)) \mathrm{d} s \\
& \geqslant \lambda \min _{t \in[\alpha, \beta]} \int_{r}^{R} G(t, s) \tilde{M} \frac{1}{2} u(s) \mathrm{d} s \\
& \geqslant \lambda \min _{t \in[\alpha, \beta]} \int_{r}^{R} G(t, s) \tilde{M} \frac{1}{4} \gamma(s) \mathrm{d} s\|u\| \\
& \geqslant \lambda \min _{t \in[\alpha, \beta]} \int_{r}^{R} G(t, s) \tilde{M} \frac{1}{4} \Gamma \mathrm{d} s\|u\| \\
& \geqslant\|u\| .
\end{aligned}
$$

This implies

$$
\|A u\| \geqslant\|u\| \quad \text { for } u \in \partial \Omega_{2} \cap K .
$$

By Theorem 1.3, $T$ has a fixed point $\tilde{u}$ with $1 \leqslant\|\tilde{u}\| \leqslant \rho_{2}$. It follows that

$$
\tilde{u}(t) \geqslant \frac{1}{2} \gamma(t) \geqslant \frac{1}{2}(2 \lambda C M) \gamma(t) \geqslant \lambda M \bar{w}(t)=w(t),
$$

and so $u=\tilde{u}-w$ is a positive solution of (1.1), completing the proof of Theorem 3.1.

\section{An example}

Let us consider the three-point BVP

$$
\left.\begin{array}{c}
u^{\prime \prime}-u+\lambda\left(u^{5}-2\right)=0, \quad 0<t<1, \\
u(0)=\frac{1}{2} u\left(\frac{1}{2}\right), \quad u(1)=\frac{1}{2} u\left(\frac{1}{2}\right) .
\end{array}\right\}
$$

Clearly, (C1) and (C2) hold. It is easy to check that

$$
\psi(t)=\frac{1}{2}\left(\mathrm{e}^{t}-\mathrm{e}^{-t}\right), \quad \phi(t)=\frac{1}{2}\left(\mathrm{e}^{1-t}-\mathrm{e}^{t-1}\right)
$$

and

Since

$$
\rho=\left|\begin{array}{cc}
\phi(0) & \psi(0) \\
\phi^{\prime}(0) & \psi^{\prime}(0)
\end{array}\right|=\left|\begin{array}{cc}
\frac{1}{2}\left(\mathrm{e}-\mathrm{e}^{-1}\right) & 0 \\
\frac{1}{2}\left(-\mathrm{e}-\mathrm{e}^{-1}\right) & 1
\end{array}\right|=\frac{1}{2}\left(\mathrm{e}-\mathrm{e}^{-1}\right) .
$$

$$
\begin{gathered}
\Delta=\left|\begin{array}{cc}
-\frac{1}{2} \psi\left(\frac{1}{2}\right) & \rho-\frac{1}{2} \phi\left(\frac{1}{2}\right) \\
\rho-\frac{1}{2} \psi\left(\frac{1}{2}\right) & -\frac{1}{2} \phi\left(\frac{1}{2}\right)
\end{array}\right|=-\rho\left(\frac{1}{2}\left(\mathrm{e}-\mathrm{e}^{-1}\right)-\frac{1}{2}\left(\mathrm{e}^{1 / 2}-\mathrm{e}^{-1 / 2}\right)\right)<0, \\
\rho-\frac{1}{2} \phi\left(\frac{1}{2}\right)=\frac{1}{2}\left(\mathrm{e}-\mathrm{e}^{-1}\right)-\frac{1}{4}\left(\mathrm{e}^{1 / 2}-\mathrm{e}^{-1 / 2}\right)>0
\end{gathered}
$$

and

$$
\rho-\frac{1}{2} \psi\left(\frac{1}{2}\right)=\frac{1}{2}\left(\mathrm{e}-\mathrm{e}^{-1}\right)-\frac{1}{4}\left(\mathrm{e}^{1 / 2}-\mathrm{e}^{-1 / 2}\right)>0,
$$

we know that $(\mathrm{C} 4)$ is satisfied. Let $\bar{w}$ be the unique solution of

$$
\left.\begin{array}{c}
u^{\prime \prime}-u+1=0, \quad 0<t<1, \\
u(0)=\frac{1}{2} u\left(\frac{1}{2}\right), \quad u(1)=\frac{1}{2} u\left(\frac{1}{2}\right),
\end{array}\right\}
$$


then

$$
\bar{w}=\frac{\left(1-\mathrm{e}^{-1}\right) \mathrm{e}^{t}+(\mathrm{e}-1) \mathrm{e}^{-t}}{2\left[\left(\mathrm{e}^{1 / 2}-\mathrm{e}^{-1 / 2}\right)+\left(\mathrm{e}-\mathrm{e}^{-1}\right)\right]}+1
$$

Moreover,

$$
\|w\|=w\left(\frac{1}{2}\right)
$$

and

$$
\|w\| \doteq 0.20334717217190629802
$$

From (2.11),

$$
\tilde{q}(t)=\min \left\{\frac{\phi(t)}{\phi(0)}, \frac{\psi(t)}{\psi(1)}\right\}=\min \left\{\frac{\mathrm{e}^{1-t}-\mathrm{e}^{t-1}}{\mathrm{e}-\mathrm{e}^{-1}}, \frac{\mathrm{e}^{t}-\mathrm{e}^{-t}}{\mathrm{e}-\mathrm{e}^{-1}}\right\}
$$

and

$$
\tilde{q}(t) \leqslant \tilde{q}\left(\frac{1}{2}\right)=\frac{1}{\mathrm{e}^{1 / 2}+\mathrm{e}^{-1 / 2}} .
$$

From (2.13) and (2.14), we know that

$$
\tilde{A}:=\frac{1}{\Delta}\left|\begin{array}{cc}
\frac{1}{2} \tilde{q}\left(\frac{1}{2}\right) & \rho-\frac{1}{2} \phi\left(\frac{1}{2}\right) \\
\frac{1}{2} \tilde{q}\left(\frac{1}{2}\right) & -\frac{1}{2} \phi\left(\frac{1}{2}\right)
\end{array}\right|=-\frac{1}{2 \Delta} \rho \tilde{q}\left(\frac{1}{2}\right)
$$

and

$$
\tilde{B}:=\frac{1}{\Delta}\left|\begin{array}{cc}
-\frac{1}{2} \psi\left(\frac{1}{2}\right) & \frac{1}{2} \tilde{q}\left(\frac{1}{2}\right) \\
\rho-\frac{1}{2} \psi\left(\frac{1}{2}\right) & \frac{1}{2} \tilde{q}\left(\frac{1}{2}\right)
\end{array}\right|=-\frac{1}{2 \Delta} \rho \tilde{q}\left(\frac{1}{2}\right) .
$$

Clearly,

$$
\tilde{A}=\tilde{B} \doteq 0.33894316655602199219 \text {. }
$$

Thus from (2.12)

$$
\begin{aligned}
\tilde{q}(t)+\tilde{A} \psi(t)+\tilde{B} \phi(t) & \leqslant \tilde{q}\left(\frac{1}{2}\right)+\tilde{A} \psi(1)+\tilde{B} \phi(0) \\
& =\tilde{q}\left(\frac{1}{2}\right)\left\{1+\frac{\rho}{-2 \Delta}\left[\frac{\mathrm{e}^{1}-\mathrm{e}^{-1}}{2}+\frac{\mathrm{e}^{1}-\mathrm{e}^{-1}}{2}\right]\right\} \\
& =\frac{1}{\mathrm{e}^{1 / 2}+\mathrm{e}^{-1 / 2}}\left\{1+\frac{\mathrm{e}^{1}-\mathrm{e}^{-1}}{\mathrm{e}-\mathrm{e}^{-1}-\mathrm{e}^{1 / 2}+\mathrm{e}^{-1 / 2}}\right\} \\
& \doteq 0.88681888397007390868
\end{aligned}
$$

So we can take $k_{0}=1$ and

$$
\gamma(t)=\tilde{q}(t)+\tilde{A} \psi(t)+\tilde{B} \phi(t)
$$

in Lemma 2.4. By (4.10)

$$
\begin{aligned}
\gamma(t) & \geqslant \tilde{A} \psi(t)+\tilde{B} \phi(t) \\
& =\tilde{A}(\psi(t)+\phi(t)) \\
& \geqslant \tilde{A} \psi\left(\frac{1}{2}\right)
\end{aligned}
$$


for all $t \in[0,1]$. This together with (4.3) imply that

$$
\bar{w}(t) \leqslant C^{*} \gamma(t), \quad t \in[0,1],
$$

where

$$
C^{*}=\frac{\|w\|}{\tilde{A} \phi\left(\frac{1}{2}\right)} \doteq 1.1513148176099288324 .
$$

Now, by the proof of Theorem 3.1, we know that (4.1) has at least one positive solution for each $\lambda \in(0, \Lambda)$ with

$$
\Lambda=\min \left\{\frac{1}{C_{1}\|\bar{w}\|}, \frac{1}{2 C^{*} M}\right\} \doteq 0.21714304044048292560
$$

where $C_{1}=1, M=2$.

Acknowledgements. The author was supported by the NSFC, GG-110-10736-1003, NWNU-KJCXGC-212 and the Foundation of Major Projects of Science and Technology of the Chinese Education Ministry. The author is very grateful to the referee for his/her valuable suggestions.

\section{References}

1. V. Anuhadha, D. D. Hai and R. Shivaji, Existence results for superlinear semipositone boundary value problems, Proc. Am. Math. Soc. 124 (1996), 757-763.

2. D. Guo and V. Lakshmikantham, Nonlinear problems in abstract cones (Academic, 1988).

3. C. P. GUPTA, Solvability of a three-point nonlinear boundary value problem for a second order ordinary differential equation, J. Math. Analysis Applic. 168 (1992), 540-551.

4. C. P. GUPTA, A generalized multi-point boundary value problem for second order ordinary differential equations, Appl. Math. Computat. 89 (1998), 133-146.

5. V. A. IL'in and E. I. Moiseev, Nonlocal boundary value problem of the first kind for a Sturm-Liouville operator in its differential and finite difference aspects, Diff. Eqns $\mathbf{2 3}$ (1987), 803-810.

6. R. MA, Positive solutions of a nonlinear three-point boundary-value problems, Electron. J. Diff. Eqns 34 (1999), 1-8.

7. M. Moshinsky, Sobre los problemas de condiciones a la frontiera en una dimension de caracteristicas discontinuas, Bol. Soc. Mat. Mexicana 7 (1950), 1-25.

8. M. H. Protter ANd H. F. Weinberger, Maximum principles in differential equations (Springer, 1984).

9. S. Timoshenko, Theory of elastic stability (McGraw-Hill, New York, 1961).

10. J. R. L. WebB, Positive solutions of some three-point boundary value problems via fixed point theory, Nonlin. Analysis 47 (2001), 4319-4332. 